ciprofloxacin resistance was first observed in the 1990s, rose sharply to more than $50 \%$ of isolates by 2008 , and has stabilised at $30-40 \%$ highlighting the ability of imported strains to become established. With recent sporadic cases of ceftriaxone-resistant gonorrhoea reported in Australia and elsewhere, we sought to model the potential for imported NG strains to persist in the men who have sex with men (MSM) population in Australia.

Methods An individual-based model was developed to represent the transmission of NG in an urban MSM population in Australia. We assume a new NG strain is imported repetitively over the course of one year into a population where NG is already endemic and examined the likelihood that an imported strain will persist for a range of importation frequencies. In doing so, we assumed that all NG strains are of similar fitness.

Results The chance that an imported strain will persist for more than 20 years is predicted to be $4 \%$ if the importation frequency is once every six months, and increases to $24 \%$ if the importation frequency is once every month. If an anatomical site can only be infected by one NG strain at a time, the model predicts that there is a $<3 \%$ chance that an imported strain will persist even if the importation frequency is once every month.

Conclusion Increasing the importation frequency increases the probability of an imported strain persisting in the population. If importation events are rare, then an imported strain is unlikely to persist unless it can coexist with local NG strains at the same anatomical site.

Disclosure of interest statement This work was supported by National Health and Medical Research Council Project Grant (APP1025517) and Program Grant (APP1071269). The Kirby Institute is funded by the Australian Government Department of Health and Ageing and is affiliated with the Faculty of Medicine, UNSW Australia. The views expressed in this publication do not necessarily represent the position of the Australian Government.

\section{P09.10 THE POTENTIAL IMPACT OF VACCINATION ON THE PREVALENCE OF GONORRHOEA}

${ }^{1} \mathrm{KL}$ Seib*, ${ }^{2} \mathrm{AP}$ Craig, ${ }^{2} \mathrm{RT}$ Gray, ${ }^{3} \mathrm{JL}$ Edwards, ${ }^{4} \mathrm{MA}$ Apicella, ${ }^{1} \mathrm{MP}$ Jennings, ${ }^{2} \mathrm{DP}$ Wilson. ${ }^{1}$ Institute for Glycomics, Griffith University; ${ }^{2}$ The Kirby Institute, UNSW; ${ }^{3}$ Center for Microbial Pathogenesis, The Research Institute at Nationwide Children's Hospital Ohio; ${ }^{4}$ Department of Microbiology, University of lowa

\subsection{6/sextrans-2015-052270.394}

Introduction Gonorrhoea, one of the most common sexually transmitted infections worldwide, can lead to serious sequelae, including infertility and increased HIV transmission. Recently, untreatable, multidrug-resistant Neisseria gonorrhoeae strains have been reported. In the absence of new antibiotics, and given the speed with which resistance has emerged to all previously used antibiotics, development of a vaccine would be the ideal solution to this public health emergency. Understanding the desired characteristics, target population, and expected impact of an anti-gonococcal vaccine is essential to facilitate vaccine design, assessment, and implementation. The modelling presented herein aims to fill these conceptual gaps and inform future gonococcal vaccine development.

Methods Using an individual-based, epidemiological simulation model, gonococcal prevalence was simulated in a heterosexual population of 100,000 individuals (with a $\sim 1.7 \%$ prevalence rate) after the introduction of vaccines with varied efficacy (10$100 \%)$ and duration of protection (2.5-20 years).

Results Model simulations predicted that gonococcal prevalence could be reduced by; at least, $90 \%$ after 20 years, if all 13 -year- olds were given a vaccine with $50 \%$ efficacy that does not wane. A comparable reduction in prevalence could be achieved by a vaccine with $100 \%$ efficacy that wanes after 7.5 years. A $40 \%$ reduction in prevalence would be achieved with a non-waning vaccine of just $20 \%$ efficacy.

Conclusion A vaccine of moderate efficacy and duration could have a substantive impact on gonococcal prevalence and disease sequelae, if coverage is high and protection lasts over the highest risk period (i.e. most sexual partner change) among youths.

Disclosure of interest statement This work was funded by the Australian National Health and Medical Research Council, The Australian Government Department of Health, and the National Institutes of Health USA. No pharmaceutical grants were received in the development of this study.

\section{P09.11 SHOULD WE SCREEN FOR MYCOPLASMA GENITALIUM? EVIDENCE SYNTHESIS USING A TRANSMISSION- DYNAMIC MODEL}

${ }^{1,2} \mathrm{R}$ Birger, ${ }^{3,4} \mathrm{~J}$ Saunders, ${ }^{3} \mathrm{C}$ Estcourt, ${ }^{5} \mathrm{AJ}$ Sutton, ${ }^{6} \mathrm{CH}$ Mercer, ${ }^{4} \mathrm{~T}$ Roberts, ${ }^{1,7,8} \mathrm{PJ}$ White ${ }^{*}$. ${ }^{1}$ MRC Centre for Outbreak Analysis \& Modelling, Department of Infectious Disease Epidemiology, School of Public Health, Imperial College London, UK; ${ }^{2}$ Department of Ecology and Evolutionary Biology, Princeton University; ${ }^{3}$ Barts and the London School of Medicine \& Dentistry, Queen Mary University of London, Barts Sexual Health Centre, UK; ${ }^{4}$ HIV \& STI Department, Centre for Infectious Disease Surveillance and Control, Public Health England, London, UK; ${ }^{5}$ University of Birmingham Health Economics Unit, UK; ${ }^{6}$ Research Department of Infection and Population Health, University College London, UK; ${ }^{7}$ NIHR Health Protection Research Unit in Modelling Methodology, Department of Infectious Disease Epidemiology, School of Public Health, Imperial College, London, UK; ${ }^{8}$ Modelling \& Economics Unit, Centre for Infectious Disease Surveillance and Control, Public Health England, London, UK

\subsection{6/sextrans-2015-052270.395}

Introduction There is increasing concern about Mycoplasma genitalium as a cause of urethritis, cervicitis, PID, infertility and ectopic pregnancy. Currently there is no licensed test specific for M. genitalium in the UK, where urethral smear microscopy is recommended in GUM clinics, for symptomatic men only. NAATs testing has been advocated, particularly to detect asymptomatic infection. However, M. genitalium's natural history is poorly-understood, making the impact and cost-effectiveness of screening unclear.

Methods We used a transmission-dynamic model to synthesise evidence from epidemiological and behavioural studies, and surveillance data for Non-Chlamydial, Non-Gonococcal Urethritis (NCNGU), to better-understand the natural history of M. genitalium. The model is stratified by sex, and incorporates heterogeneous sexual behaviour, symptomatic and asymptomatic infection; PID; care-seeking due to symptoms and routine screening; and treatment failure. We fitted to national surveillance data, (allowing for uncertainty in studies measuring the amount of NCNGU caused by M. genitalium). We examined the effects of implementing NAAT testing for both sexes in GUM and GP settings.

Results Introducing NAAT testing for all men (asymptomatic and symptomatic, in GPs and GUM) detects much more infection in men and treatment reduces transmission to women, whilst testing of women reduces prevalence in women and incidence in men. Introducing NAAT testing for both sexes reduces cumulative PID incidence over 20 years by $13.1 \%$ (IQR:9.6\%$18.3 \%)$. However, there is important uncertainty in M. genitalium's natural history parameters, leading to uncertainty in the absolute reduction in PID and other sequelae. Particularly 
important are the proportion of infections that are symptomatic in men and women; duration of untreated infection; and incidence of PID, infertility and ectopic pregnancy attributable to M. genitalium.

Conclusion Further empirical work is required to improve understanding of the key aspects of M. genitalium's natural history which we have identified before it will be possible to determine if screening is cost-effective.

Disclosure of interest statement This presentation reports independent research commissioned by the National Institute for Health Research (NIHR) under its Programme Grants for Applied Research scheme (RP-PG-0707-10208). In addition, PJW thanks the UK Medical Research Council for Centre funding (grant MR/K010174/1) and also thanks the UK National Institute for Health Research Health Protection Research Unit (NIHR HPRU) in Modelling Methodology at Imperial College London in partnership with Public Health England (PHE) for funding (grant HPRU-2012-10080). The views expressed in this paper are those of the authors and not necessarily those of the NHS, the NIHR, the Department of Health, or Public Health England. The funders had no role in study design, data collection and analysis, decision to publish, or preparation of the manuscript. We have no conflicts of interest.

\section{P09.12 APPARENTLY-DIFFERENT CLEARANCE RATES FROM COHORT STUDIES OF MYCOPLASMA GENITALIUM ARE CONSISTENT AFTER ACCOUNTING FOR INCIDENCE OF (RE) INFECTION AND STUDY DESIGN}

${ }^{1,2} \mathrm{~T}$ Smieszek, ${ }^{1,2} \mathrm{PJ}$ White* ${ }^{1}$ Modelling and Economics Unit, Centre for Infectious Disease Surveillance and Control, Public Health England, London, UK; ${ }^{2}$ NIHR Health Protection Research Unit in Modelling Methodology and MRC Centre for Outbreak Analysis and Modelling, Department of Infectious Disease Epidemiology, School of Public Health, Imperial College London, UK

\subsection{6/sextrans-2015-052270.396}

Introduction Mycoplasma genitalium is increasingly recognised as an important cause of urethritis, cervicitis, PID, infertility, and increased HIV risk. A better understanding of its natural history is crucial to informing control policy. Two cohort studies (students in London, UK, and sex workers in Uganda) suggest very different clearance rates. We aimed to gain insight into the reasons and to obtain improved estimates by making maximal use of the data from those studies.

Methods To estimate incidence and clearance rates, we developed a model for time-to-event analysis incorporating the processes of individuals becoming infected after enrolment into the study, clearing infection, becoming reinfected after clearance, and fitted it to data from the two cohort studies. As the studies collected limited data on sexual partnership dynamics, we tested the sensitivity of the model to different assumptions that were consistent with the available information. Additionally, we modelled study-design differences, including sample handling conditions affecting testing sensitivity.

Results In the London students, the estimated clearance rate was 0.80p.a. (mean duration 15 months), with incidence $1.31 \%$ p.a. and $3.93 \%$ p.a. (in low- and high-risk groups, respectively). Without adjusting for study design, corresponding estimates from the Ugandan data were 3.35p.a. (mean duration 3.6 months) and $44 \%$ p.a. Clearance-rate differences could be explained by differences in testing sensitivity, with 'true' rates being similar, and adjusted incidence in the Uganda study being $21 \%$ p.a.
Conclusion Analysis needs to account for study design, and we recommend cohort studies collect more information on partnership dynamics to inform more-accurate estimates of natural-history parameters. The cohorts' clearance rates were probably similar, with the apparent difference due mostly to differences in sample handling in the studies, and perhaps partly due to the sex workers having more-frequent antibiotic treatment (for other infections), and in the London students some reinfection in stable partnerships causing some of the apparently-persistent infection.

Disclosure of interest statement TS and PJW thank the UK National Institute for Health Research Health Protection Research Unit (NIHR HPRU) in Modelling Methodology at Imperial College London in partnership with Public Health England (PHE) for funding (grant HPRU-2012-10080). PJW also thanks the UK Medical Research Council for Centre funding (grant MR/K010174/1). The views expressed are those of the authors and not necessarily those of the NHS, the NIHR, the Department of Health, or Public Health England. We have no conflicts of interest.

\section{P09.13 IMPACT AND COST-EFFECTIVENESS OF POINT-OF-CARE TESTING FOR CHLAMYDIA: ACCOUNTING FOR GEOGRAPHIC VARIATION IN INFECTION BURDEN AND TESTING RATES, HEALTH SERVICE CONFIGURATION, AND IMPLEMENTATION STRATEGY}

${ }^{1,2,3,4} \mathrm{CE}$ Dangerfield, ${ }^{1,2,3} \mathrm{E}$ Sherrard-Smith, ${ }^{2,3} \mathrm{~N}$ Green, ${ }^{1} \mathrm{E}$ Harding-Esch, ${ }^{1} \mathrm{R}$ Howell-Jones, ${ }^{5}$ Y Choi, ${ }^{1,6} \mathrm{CM}$ Lowndes, ${ }^{2,3} \mathrm{PJ}$ White*. ${ }^{1}$ HIV \& STI Department, Centre for Infectious Disease Surveillance and Control, Public Health England, London, UK; ${ }^{2}$ Modelling \& Economics Unit, Centre for Infectious Disease Surveillance and Control, Public Health England, London, UK; ${ }^{3}$ MRC Centre for Outbreak Analysis and Modelling and Health Protection Research Unit in Modelling Methodology, Department of Infectious Disease Epidemiology, Imperial College London, UK; ${ }^{4}$ Department of Plant Sciences, University of Cambridge, UK; ${ }^{5}$ Immunisation Department, Centre for Infectious Disease Surveillance and Control, Public Health England, London, UK; ${ }^{6}$ London School of Hygiene and Tropical Medicine, UK

\subsection{6/sextrans-2015-052270.397}

Introduction Point-of-care tests (PoCTs) for Chlamydia trachomatis potentially improve control by reducing transmission through reduced treatment delay, reduced loss to follow up, and greater convenience for patients leading to increased testing. However, with $\sim 3-5$ fold variation in rates of Chlamydia testing and diagnosis across geographic settings in England, in assessing cost-effectiveness the epidemiological context needs to be considered.

Methods We developed a transmission-dynamic model to capture geographic variation in rates of testing and diagnosis using current technology to allow assessment of the impact of implementing PoCTs in different clinical services in different localities. The model incorporates heterogeneity in sexual partner change rates and is stratified by age and sex. It uses behavioural and prevalence data from the Natsal national survey, and Public Health England surveillance data on testing and diagnosis rates. Uncertainty in natural history and behavioural parameters is captured by Monte Carlo methods. Health service reconfiguration using PoCT is considered, including rates of PoCT introduction and reduction in presumptive treatment.

Results The model captures observed geographic variation in rates of testing and diagnosis in females and males, which affects the impact and cost-effectiveness of PoCT introduction. In general, whilst PoCTs may reduce incidence by increasing diagnosis 